\title{
Reduced brain resting-state network specificity in infants compared with adults
}

\author{
This article was published in the following Dove Press journal: \\ Neuropsychiatric Disease and Treatment \\ 21 July 2014 \\ Number of times this article has been viewed
}

\author{
Korey P Wylie ${ }^{1, *}$ \\ Donald C Rojas',* \\ Randal G Ross' \\ Sharon K Hunter' \\ Keeran Maharajh' \\ Marc-Andre Cornier ${ }^{2}$ \\ Jason R Tregellas ${ }^{1,3}$ \\ 'Department of Psychiatry, ${ }^{2}$ Division \\ of Endocrinology, Metabolism and \\ Diabetes, Department of Medicine, \\ University of Colorado Anschutz \\ Medical Campus, Aurora, CO, USA; \\ ${ }^{3}$ Denver Veterans Affairs Medical \\ Center, Denver, CO, USA \\ *These authors contributed equally \\ to this work
}

Purpose: Infant resting-state networks do not exhibit the same connectivity patterns as those of young children and adults. Current theories of brain development emphasize developmental progression in regional and network specialization. We compared infant and adult functional connectivity, predicting that infants would exhibit less regional specificity and greater internetwork communication compared with adults.

Patients and methods: Functional magnetic resonance imaging at rest was acquired in 12 healthy, term infants and 17 adults. Resting-state networks were extracted, using independent components analysis, and the resulting components were then compared between the adult and infant groups.

Results: Adults exhibited stronger connectivity in the posterior cingulate cortex node of the default mode network, but infants had higher connectivity in medial prefrontal cortex/anterior cingulate cortex than adults. Adult connectivity was typically higher than infant connectivity within structures previously associated with the various networks, whereas infant connectivity was frequently higher outside of these structures. Internetwork communication was significantly higher in infants than in adults.

Conclusion: We interpret these findings as consistent with evidence suggesting that restingstate network development is associated with increasing spatial specificity, possibly reflecting the corresponding functional specialization of regions and their interconnections through experience.

Keywords: functional connectivity magnetic resonance imaging, networks, development, infancy, resting-state, connectivity

\section{Introduction}

Recent theories of brain development in children have predicted that brain development would be characterized by increasing functional specialization with age. For example, Johnson ${ }^{1,2}$ has proposed the Interactive Specialization (IS) framework, which predicts that networks will become more specialized or segregated as they mature, and likewise, that regions within networks will become functionally more specialized through experience (ie, hierarchical scaling of specialization). IS theory is broadly similar to Werner's “orthogenetic principle" of development, articulated more than half a century ago, that child development could be characterized by increasing differentiation and hierarchical organization of behavior. ${ }^{3}$ Although this perspective is common in the classical literature on child development, it has only more recently been articulated within developmental neuroscience.

The developmental functional specialization of the human brain is relatively poorly understood from the perspective of neuroimaging. Recent work in functional connectivity magnetic resonance imaging (MRI) has, however, opened a new venue for studying the functional specificity of infant brain networks. Resting-state networks
Correspondence: Jason R Tregellas Department of Psychiatry, Box F456, University of Colorado Anschutz Medical Campus, I300I E 17th Ave, Aurora, CO, USA

Tel $+\mathrm{I}-303-724-4420$

$\mathrm{Fax}+\mathrm{I}-303-724-6227$

Email jason.tregellas@ucdenver.edu submit your manuscript | www.dovepress.com

Dovepress

http://dx.doi.org// 0.2147/NDT.S63773
Neuropsychiatric Disease and Treatment 2014:10 1349-1359 
(RSNs) are spatially distinct regions of the brain that exhibit a low-frequency temporal coherence. Also called intrinsic networks, RSNs have been reasonably well-characterized in healthy adults. ${ }^{4}$ More recent work has also examined RSN activity in preterm and full-term infants ${ }^{5}$ (reviewed in Vogel et al), ${ }^{6}$ identifying some RSNs grossly similar to those of adults. At least one study has also observed RSN activity from human fetuses in utero. ${ }^{7}$

One of the more striking perinatal RSN findings is that preterm and full-term infants do not exhibit the same coherent spatial distribution of the default mode network (DMN). In the perinatal period, the medial prefrontal and precuneus nodes of the DMN are often found in separate components in independent components analysis (ICA), unlike in adults. ${ }^{8-14}$ This has led some investigators to term the DMN-like RSN, "proto-DMN."10,11

Other RSNs separate from DMNs are consistently identified in preterm and newborn infants, using functional connectivity MRI, including auditory/temporal, sensorimotor, visual, subcortical/basal ganglia, and cerebellar networks. ${ }^{5,8-11}$ Executive/cognitive control networks have also been identified in late-preterm and full-term infants. ${ }^{9}$ One finding from RSN developmental studies is the transition away from higher short-distance connectivity toward higher long-range connectivity with age. For example, the mean distance between anatomical regions exhibiting greater connectivity in children than adults is reportedly shorter than those connections exhibiting higher connectivity in the adults. ${ }^{15}$ A similar finding was reported by Fair et $\mathrm{l}^{16}$ who found an increase in long-range connectivity with increasing age. Decreasing local or adjacent connectivity is a finding consistent with the specialization predictions of the IS framework.

The current study examines RSNs directly between infants and adults, employing ICA to decompose a set of RSN components common to both groups for comparison. On the basis of prior work on infant RSNs, and in line with IS theory, ${ }^{2}$ we predicted that infant resting-state functional connectivity would exhibit less spatial specificity within each network (eg, for the auditory RSN, that auditory activity in infants would be reduced in canonical auditory regions compared with in adults). We also hypothesized that greater between-RSN correlations would be observed in infants, a prediction that also emerges directly from the IS framework. Last, we hypothesized that the decreased spatial specificity of infant RSNs will be reflected in regions that, although in adult brains are associated with a single network, in infant brains will show nonspecific connections with many RSNs.

\section{Material and methods Subjects}

Fourteen infants participated in the study. All were healthy, term deliveries with unremarkable prenatal histories. Of the 14 infants, two were excluded from analyses because of excessive movement during the functional connectivity MRI scans ( $>1$ voxel). The remaining 12 infants (seven girls) were $55.86 \pm 20.3$ days old at the time of the scan (mean age adjusted for gestational age at birth on day of scan, $50.57 \pm 21.79$ days). Seventeen adults also participated in the study (eight women; mean age, 30.82 \pm 2.63 years). Adult subjects and parents of the infants provided written informed consent consistent with the guidelines of the local institutional review board and the Declaration of Helsinki. The Colorado Multiple Institutions Review Board approved this study.

\section{MRI methods}

A General Electric (Milwaukee, WI, USA) 3T Signa MRI system was used to acquire MR data. Infant scans were performed immediately after feeding by a parent. Infants were swaddled and slept unsedated during scans. One hundred and fifty $\mathrm{T} 2 *$ echo-planar imaging (EPI) volumes were acquired (repetition time [TR] $=2,000 \mathrm{~ms}$, echo time $[\mathrm{TE}]=30 \mathrm{~ms}$, field of view $=22 \mathrm{~cm}^{2}$, flip angle $=70^{\circ}$, matrix $=64^{2}, 28$ axial, $3.4 \mathrm{~mm}$ thick slices, no gap, angled parallel to the planum sphenoidale), using a standard quadrature head coil. Voxel dimensions were $3.43 \times 3.43 \times 3.4 \mathrm{~mm}$. Slices were $2.6 \mathrm{~mm}$ thick, with a $1.4 \mathrm{~mm}$ gap for the adult scan data $(\mathrm{N}$ slices $=27$; dimensions $=3.43 \times 3.43 \times 4 \mathrm{~mm}$ ). Motion was reduced using foam inserts between the head and coil. Headphones were used for hearing protection during scans. Subsequent to the EPI acquisition, inversion-recovery $\mathrm{T} 1$ weighted scans were acquired for each participant $(\mathrm{TR}=6.56 \mathrm{~ms}, \mathrm{TE}=2.82 \mathrm{~ms}$, inversion recovery time $[\mathrm{IR}]=450 \mathrm{~ms}$, flip angle $=12^{\circ}, 122$ axial slices, $1 \mathrm{~mm}^{3}$ voxels).

\section{Data analysis}

The first four EPI scans were excluded from analysis to ensure T1 saturation. Data were preprocessed using Statistical Parametric Mapping 8 (SPM8; Wellcome Department of Imaging Neuroscience, London, UK), including reslicing to $3 \mathrm{~mm}^{3}$, motion correction/realignment to the first image volume, normalization to the Montreal Neurological Institute EPI template included in SPM8, and smoothing using an $8 \mathrm{~mm}$ full width at half maximum Gaussian kernel. Data from the T1 scans were not used for coregistration or normalization because of the poor quality of gray and 
white matter contrast in the infant data sets. Additional motion correction, after prior steps including realignment, was carried out using independent components analysis by excluding artifactual components from the analysis. ${ }^{17}$

The Group ICA of fMRI (GIFT) Toolbox (http://icatb. sourceforge.net) was used to conduct group Independent Components Analysis, using data from both groups to estimate components. The minimum description length criterion was calculated for each subject, and the mean minimum description length set the maximum number of ICA components. Data from all subjects in both groups were concatenated and reduced to 30 dimensions, using principal component analysis. This was followed by ICA decomposition, using the infomax algorithm. ${ }^{18}$ Individual component maps and time courses were back-reconstructed, using the GICA3 (PCA-based direct back-reconstruction approach group ICA method 3) option and converted to $z$-scores. Other GIFT options followed the specifications in Allen et al. ${ }^{19} \mathrm{~A}$ second ICA using identical parameters was carried out on the infant subjects alone. Regression-based spatial sorting was then used to compare components from the infant-only and the combined ICA. Additional regression-based spatial sorting to adult RSN templates ${ }^{20}$ was used to identify ICA components that resembled reported RSNs.

For each component, to ensure later group differences would be restricted to component regions, a mask was applied for subsequent analysis by performing a one-sample Student's $t$-test across the infant and adult samples and thresholding the SPM, using a correction for multiple comparisons at FWE (familywise error rate $)=0.05$. These masked components were used for second-level statistical analyses between groups. Group comparisons (adult $>$ infant and infant $>$ adult) were performed using independent Student's $t$-tests in SPM8, assuming unequal variance. Results were height-thresholded at FWE $P=0.05$, with an extent threshold of 5 voxels, to control for multiple comparisons.

For between-RSN correlations, partial correlation was used to measure connectivity between components. This accounts for indirect influences on the covariance between pairs of components by projecting their time courses onto a subspace orthogonal to all other components. ${ }^{21}$ Subjectspecific time courses for each component were extracted, and the partial correlation matrix was calculated using all components for each subject. ${ }^{2}$ Because we were concerned only with the magnitude of the correlations, their absolute value was used for further statistical analysis. The mean absolute correlation coefficient between all matched components was calculated for each subject and used in a Student's $t$-test between groups. To compare the connectivity of compo- nents, the sum of the absolute correlation coefficients was calculated for each component and used in a Student's $t$-test between groups.

To visually demonstrate regions with nonspecific higher-connectivity between the groups, we used statistical parametric networks ${ }^{23}$ at select points. For each voxel, we constructed a mean connectivity vector by averaging ICA weights over all subjects within each group. Vectors for each group were compared using Pearson's correlation, with negative correlations interpreted as dissimilarity between groups. The three clusters of dissimilarity within gray matter voxels were identified, and their connectivity to ICA components was displayed as weighted bipartite statistical parametric networks. Briefly, each ICA weight for these voxels was converted into $z$-scores, thresholded at $P<0.05$ (false discovery rate [FDR] corrected), ${ }^{24}$ and displayed on sagittal, coronal, and axial glass brain silhouettes.

\section{Results}

We first determined whether combining infant and adult groups into a single ICA resulted in differences in the networks isolated compared with ICA using the infant subjects alone. Within the same group of pediatric subjects, a similar set of infant RSNs were identified, whether or not adult subjects were included in the analysis (Figure S1). Visual inspection of the resulting networks revealed only minor differences. The infant-only ICA isolated separate anterior and posterior components of the dorsal DMN compared with a single dorsal DMN component in the combined ICA. In addition, the infant-only ICA included a single component for the cerebellum. All networks in the combined ICA correlated highly with at least one network in the infant-only ICA (mean $r_{\text {max }}=0.54$, standard deviation $[\mathrm{SD}]=0.18$ ). These results suggest that the inclusion of adults in the combined ICA had minimal influence on the analysis and that comparable RSNs exist in both infants and adults.

RSNs commonly identified in adult studies provide a convenient starting point for analyzing infant networks, but their suitability for infant networks is unknown. We separated the infant and adult spatial maps into separate groups and correlated their respective mean spatial maps to established RSN templates. ${ }^{20}$ In both cases, as well as in the combined case, spatial sorting identified almost the same set of components for top matches with the RSN templates. Two RSN templates, referred to as the posterior salience network and the ventral DMN, matched inconsistently across groups and weakly in all cases $\left(r_{\max }<0.2\right)$. After excluding these networks, all remaining correlations with template RSNs were substantial $\left(r_{\max }>0.2\right)$ and relatively 
robust across groups (mean $r_{\max }=0.4$ [SD $\left.=0.15\right]$ in adults; mean $r_{\text {max }}=0.3$ [SD $=0.12$ in infants]). Similar correlations resulted from comparing infant-only ICA with the same RSN templates (mean $r_{\max }=0.3 ; \mathrm{SD}=0.08$ ). These results suggest the adult RSN templates can be used to analyze infant networks and that differences exist between adult and infant RSNs.

On the basis of the similarities between these results, all subsequent analyses were carried out using subject-specific spatial maps from the combined ICA to directly compare equivalent RSNs in both groups. Results of group comparisons for components associated with DMN and other RSNs are illustrated in Figures 1 and 2, respectively. Table S1 provides details of the group comparisons, including Montreal Neurological Institute coordinates for all clusters surviving multiple comparison corrections at FWE $<0.05$. These findings listed in the supplementary materials are also briefly summarized in the following sections.

\section{DMNs}

An RSN consisting of the medial prefrontal cortex (mPFC) and precuneus/posterior cingulate cortex, angular gyri, and hippocampi matched the template for the dorsal DMN. ${ }^{4}$ Within this network, the adult group exhibited significantly increased functional connectivity relative to infants within the left and right precuneus and left posterior cingulate gyrus. Infants exhibited significantly higher connectivity in the right $\mathrm{mPFC}$, right anterior cingulate gyrus, and right superior frontal gyri relative to adults. Because the "ventral" DMN matched different networks in either group, we considered this template to be unreliable for comparing adult with infant RSNs.

\section{Primary sensory networks}

Several networks showed distributions that included primary sensory regions in both groups. A network consisting of the bilateral superior temporal gyrus matched the template for the auditory network. The adult group had significantly higher connectivity within right anterior superior temporal gyrus (STG), whereas infants had higher connectivity in the right posterior STG and left STG/supramarginal gyrus.

Another network, centered on bilateral calcarine sulci, matched the template for the primary visual RSN. Within this network, adults had higher connectivity in the left and right calcarine sulci, whereas infants had higher connectivity in a single cluster consisting of the left fusiform and lingual gyri.

Bilateral regions around the central sulci formed a network that matched with the sensorimotor RSN template. Adults exhibited higher connectivity in the right precentral gyrus, whereas infants had higher connectivity than adults in a cluster consisting of right superior parietal and right postcentral gyrus regions.

\section{Insula and dorsal cingulate salience network}

A network consisting of anterior portions of the left and right insula, bilateral middle frontal gyri, dorsal anterior cingulate cortex, mPFC, and bilateral Crus I regions of the cerebellum

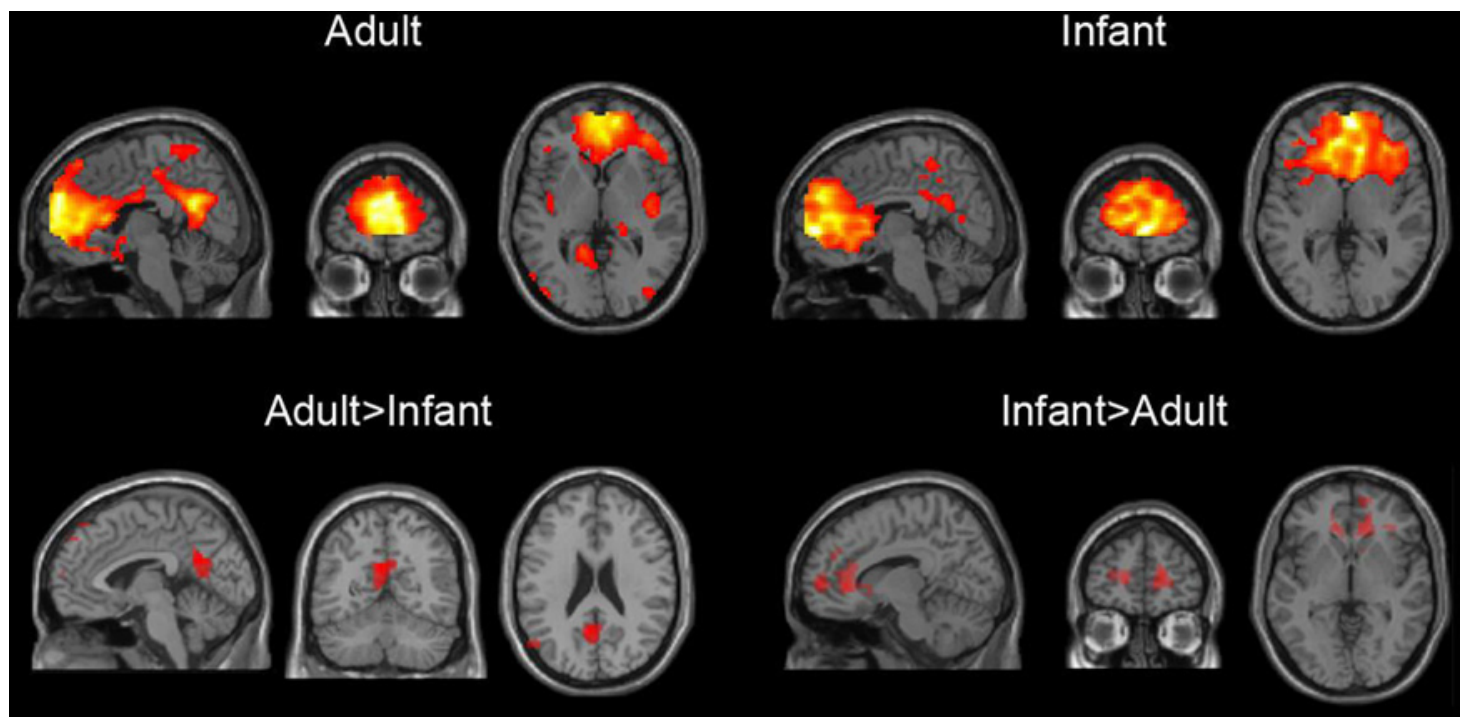

Figure I Default mode network in adults and infants.

Notes: Each triplet shows the same brain in sagittal, coronal, and axial views. The top row reflects regional associations with the default mode network within each age group, from one-sample Student's $t$-tests at an uncorrected $P<0.00 \mathrm{I}$ threshold. The bottom row illustrates regional differences between the groups within the same default mode network component, shown as adults greater than infants (left) and infants greater than adults (right), FWE-corrected for multiple comparisons at $P<0.05$.

Abbreviation: FWE, familywise error rate. 


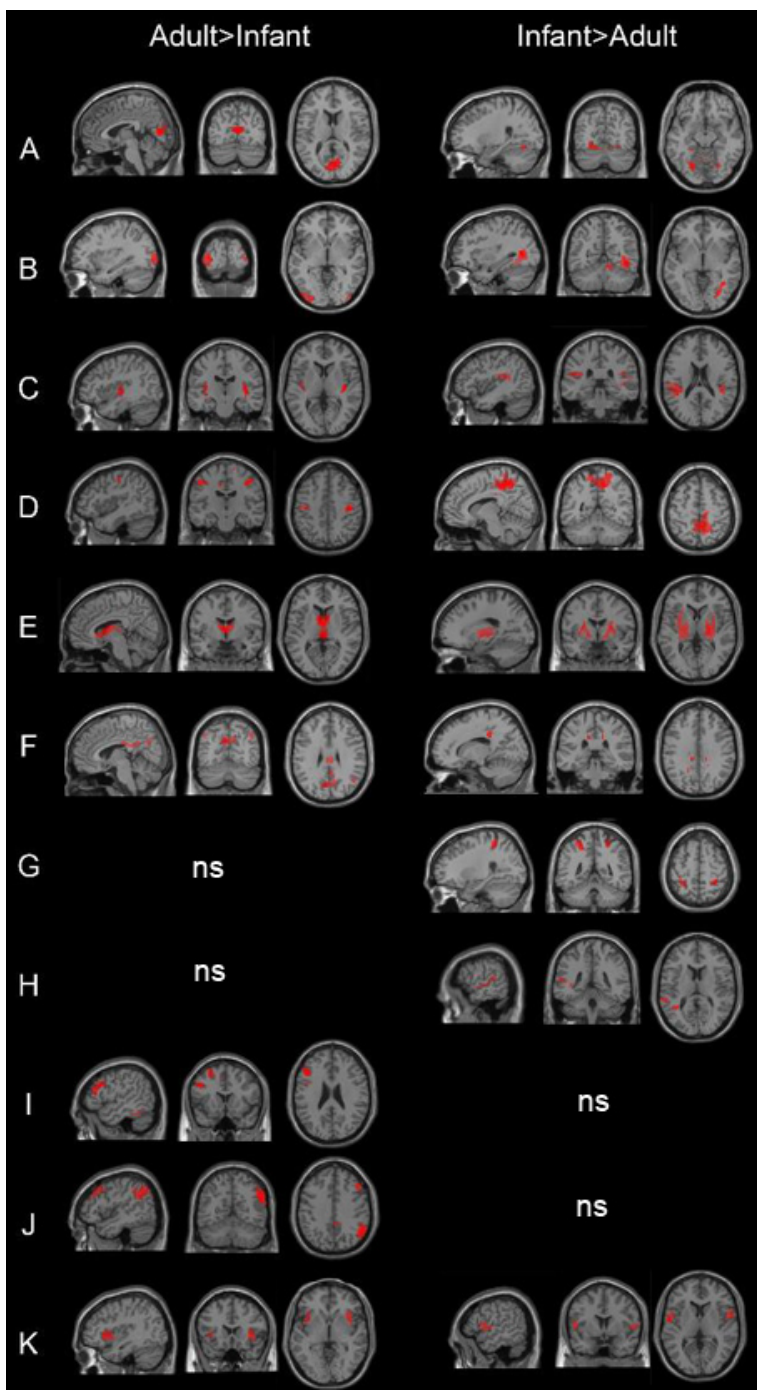

Figure 2 Regional differences in resting-state networks in infants and adults. Notes: Shown are regions that differ in their association to the same network between adults and infants. Each brain is shown in sagittal, coronal, and axial views and is displayed in two columns based on the direction of the group contrast, with the adults greater than infants on the left and infants greater than adults on the right. All contrasts thresholded at $P<0.05 \mathrm{FWE}$-corrected for multiple comparisons. Resting-state network labels: (A) VI, (B) V2, (C) auditory, (D) sensorimotor, (E) basal ganglia, (F) precuneus, (G) visuospatial, (H) language, (I) left executive control, (J) right executive control, and (K) anterior salience.

Abbreviations: ns, nonsignificant; FWE, familywise error rate.

matched the anterior salience network template. Adults had higher connectivity in left and right insula within this network, as well as the right supramarginal gyrus. Infants had higher connectivity than adults in bilateral rolandic operculum and right midcingulate gyrus.

\section{Dorsolateral prefrontal and parietal networks}

Two networks, both consisting of the dorsolateral prefrontal cortex (DLPFC) and superior parietal lobule in adults and infants, matched templates for the left and right executive control networks. For the left DLPFC/parietal network, the adults exhibited higher functional connectivity in the left inferior temporal gyrus, as well as the left middle frontal gyrus (DLPFC). For the right DLPFC/parietal network, adults also exhibited higher right middle frontal gyrus connectivity, as well as within the right superior frontal gyrus, middle cingulum, and right parietal cortex, including angular and supramarginal gyri, compared with infants. Infants did not have any regions exhibiting higher connectivity than adults for the left or right DLPFC/parietal RSNs.

\section{Temporal and frontal language network}

A network composed of the regions around the temporal sulcus and bilateral inferior frontal gyrus matched the language network template. The adults did not exhibit any significantly higher connectivity than the infants in this network. Infants, however, had significantly higher connectivity in the left superior temporal gyrus compared with adults.

\section{Possible infant-specific networks}

The ICA algorithm may interpret differences between adult and infant brains by identifying networks that are primarily associated with one group, with minimal spatial distributions in the other. To test this possibility, we examined all networks without RSN template matches in a manner similar to the networks detailed earlier.

A small number of networks were predominantly found in infants, with minimal distributions in adults (Table S1). One such network, labeled ICA component 14, featured clusters around the anterior insula/temporal poles bilaterally and the medial orbitofrontal cortex. In group contrasts, these same three areas showed increased connectivity in infants. No region was significantly increased in adults. Another network, component 18, consisted of bilateral clusters in the DLPFC and inferior parietal lobes. Group comparisons showed distinct anterior or posterior distributions in either group. Adults, compared with infants, had increased connectivity in the left DLPFC. Infants, compared with adults, had increased connectivity in bilateral inferior parietal clusters.

\section{Internetwork connectivity}

Reduced segregation in infant RSNs, as predicted by IS framework, may involve increased temporal overlap as well, suggesting there will be increased correlations between these networks in infants compared with in adults. Partial correlation analyses confirmed this and revealed that infants had increased overall functional connectivity between networks in comparison with adults (t[29] $=5.15 ; P=0.0001)$. This finding did not appear to be driven by any particular network. Virtually all pairs of RSNs exhibited increased connectivity 
in infants compared with adults. No individual correlation between networks survived FDR correction at $P<0.05$.

\section{Infant brain regions that connect RSNs}

The ability to mediate information flow between RSNs is associated with regions within the brain that are strongly connected to many large-scale networks. ${ }^{25}$ Because these regions show nonspecific connectivity that bridges multiple segregated networks, IS framework predicts there will be more of these areas in infants compared with adults. We investigated this possibility by searching for voxels with dissimilar voxel-to-RSN connectivity profiles, between adults and infants, and displaying their connections via statistical parametric networks. ${ }^{23} \mathrm{We}$ identified three clusters with dissimilar higher-level connectivity in the bilateral parahippocampal gyri and right amygdala (Figure 3). In adult brains, the parahippocampal clusters were significantly connected to a single network, whereas the cluster within the amygdala was connected to a second network. In contrast, in infants, these same clusters exhibited widespread and stronger connectivity to many RSNs.

\section{Discussion}

A general pattern of connectivity observed in various RSNs, including networks encompassing primary sensory regions, was that adults showed increased connectivity within the component in regions of primary sensory or motor cortex (eg, calcarine sulcus for the primary visual network), whereas infants exhibited higher connectivity outside of the primary regions within the same network (eg, the fusiform and lingual gyri for the primary visual network). In the auditory network, adults exhibited higher connectivity in Heschl's gyrus than the infants, whereas infants exhibited stronger responses in posterior portions of the STG, nominally associated with secondary auditory cortex and in the supramarginal gyrus, part of a polymodal association region in the parietal lobe. In the sensorimotor network around the central sulci, this pattern also was evident, with adult connectivity higher in the precentral gyrus, whereas infant connectivity was higher outside of typical somatomotor regions, including a large cluster centered in the precuneus and extending to superior parietal lobule and postcentral gyrus. This finding was not restricted to sensory and motor networks, as the insula and cingulatecentered salience network also exhibited more connectivity in infants outside of the dorsal anterior cingulate cortex and bilateral insula compared with adults. We also observed a similar pattern in at least part of the DMN: Adults had higher functional connectivity in posterior cingulate cortex than infants, and infants had higher functional connectivity in the anterior mPFC node than adults. The greater spatial extent of infant $\mathrm{mPFC} /$ anterior cingulate cortex functional connectivity is consistent with previous findings in infants ${ }^{14}$ and adolescents ${ }^{26}$ compared with adults.
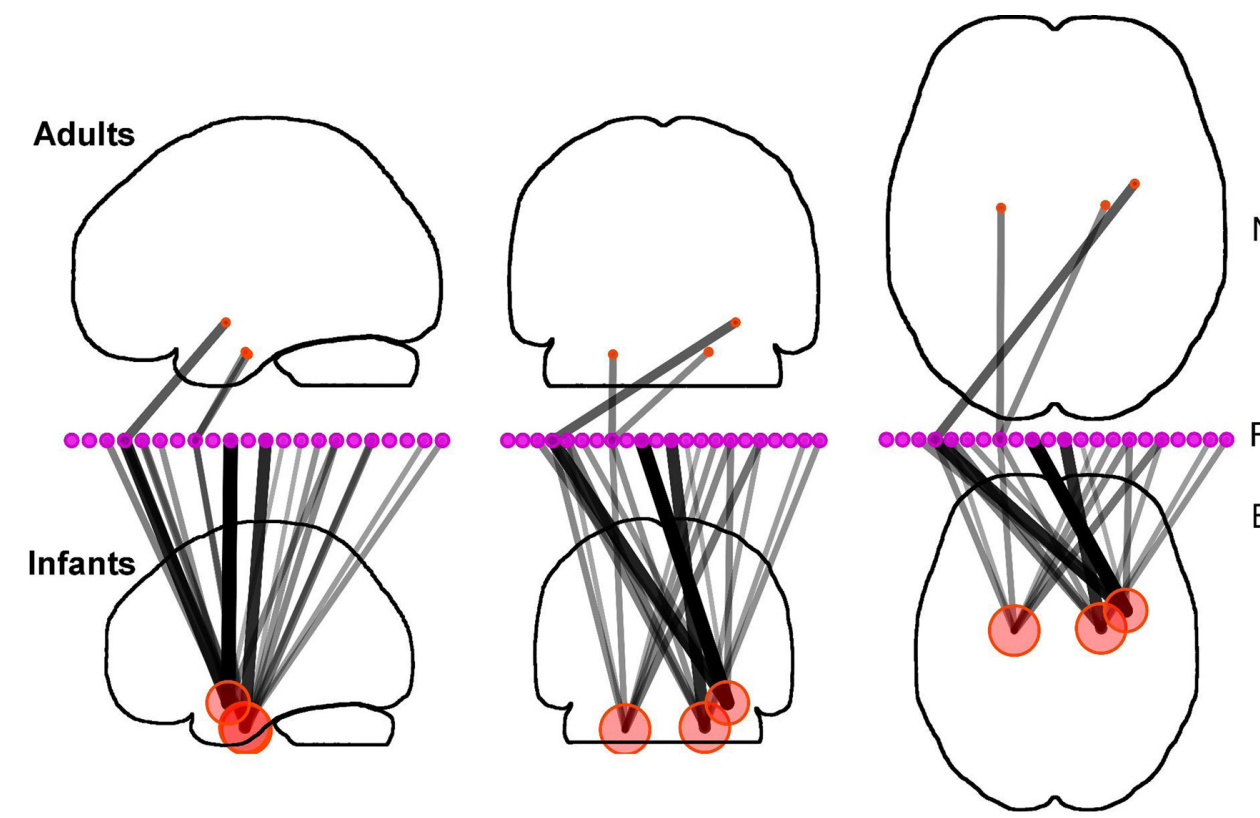

Nodes (voxel degree):

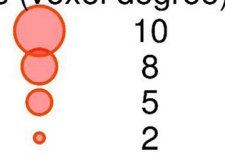

Resting-state networks: ○००० (RSN nodes) Edges (ICA z-score):

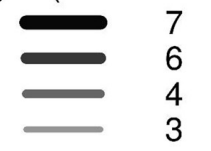

Figure 3 Higher-level connectivity in infants and adults.

Notes: The connectivity between resting-state networks (independent components analysis, displayed as purple circles) differed between infants and adults within three clusters (red circles), located with the bilateral parahippocampal gyri and right amygdala. Each location in adults was significantly connected to a single resting-state network, whereas infants featured both stronger and less-specific connectivity. All connections thresholded at FDR-corrected $P<0.05$.

Abbreviations: RSN, resting-state network; ICA, independent components analysis; FDR, false discovery rate. 
The overall results are therefore consistent with a pattern of spatially more restricted local connectivity in mature adult RSNs, possibly indicative of more functional specialization with greater maturity. It was not a ubiquitous observation, however, as, for example, within the frontoparietal executive control networks, there were no areas in which infant group connectivity exceeded that of the adult group. In contrast, adults had higher connectivity evident in both left and right frontal and posterior components of the executive networks. Also, within the language network, infants were observed to have greater connectivity within the left STG, certainly a region classically associated with language function, whereas adults had no language network areas of significantly greater connectivity.

Overall, we also observed that between-network connectivity was increased in the infant group relative to the adults, as measured by inter-RSN partial correlation coefficients. A similar finding was reported by Stevens et a ${ }^{27}$ who reported on internetwork interactions in a sample of 100 healthy individuals aged $12-30$ years. The authors found a significant inverse correlation between age and internetwork interactions. Although the most dramatic changes occurred during early adolescence, there was also evidence for continued development past age 30 years, given the lack of evidence for an asymptote in internetwork interactions. Taken together with the current findings, this suggests that internetwork communication is highest in early development and continues to develop well into at least the early adult years. At the same time, evidence strongly suggests that within a given network, children tend to exhibit reduced interregional connectivity compared with adults, ${ }^{9}, 14,20,28$ which is consistent with a developmental framework of increasing segregation and compartmentalization of brain regions and networks through experience. IS theory predicts this type of developmental progression. ${ }^{1}$

A potential limitation of this study is that there are currently no RSN templates available based on infant studies. Our finding of networks found predominantly in infants argues for the need for such templates. To overcome this potential limitation, we have used the RSN templates purely as useful descriptions of previously identified RSNs. The relatively high correlations between the RSN templates and infant networks suggest this comparison is at least partially valid. In all cases, we have deliberately avoided limiting our analysis only to networks associated with these templates. We examined every ICA component using the same techniques, regardless of whether or not they resembled an RSN template.

Another important limitation for the current study is that we obtained no behavioral correlates associated with the differences in RSNs. Although our results are consistent with the IS framework, the proposed mechanism through which specialization occurs in development is perceptual experience, which cannot easily be assessed without a taskbased approach. Simple sensory stimuli can be delivered effectively in sleeping or sedated infants, an approach that would yield a more direct test of IS framework predictions concerning functional specialization. For example, Heep et $\mathrm{al}^{29}$ reported bilateral activation of sensorimotor regions to unilateral stimulation in a study of eight sedated, preterm infants, interpreting the bilateral activation as a marker of brain immaturity. Studies combining sophisticated behavioral and neuroimaging methodologies are needed to assess the effect of functional segregation and specialization on cognitive development in young children.

\section{Conclusion}

We observed two main patterns of results in the infant data compared with adults. First, within several networks, infants had greater functional connectivity outside of the regions considered to be canonical network structures in the adult brain (eg, primary sensory areas in primary sensory networks), whereas adults exhibited higher connectivity within the canonical structures. This pattern was not observed for all networks, however, including the language and executive networks. Second, between-RSN connectivity was higher in the infants than the adults. We interpret these findings within the developmental context that functional specialization increases the spatial segregation and specificity within a network and that network activity also becomes more segregated. These predictions emerge directly from the IS and similar developmental specialization theories. Specific studies of behavioral and cognitive skill development, concurrent with measures of brain network activity, will need to be conducted to directly test hypotheses emerging from the IS framework, however.

\section{Acknowledgments}

Support for this research came from National Institutes of Health grants R01 MH082820, R01 MH081920, R01 DK089095, and P50 MH086383; the Brain Research Foundation; and the Blowitz-Ridgeway Foundation. The financial sponsors had no role in the design, data collection analysis, data interpretation, or writing of the manuscript or decision to submit the manuscript for publication.

\section{Disclosure}

The authors report no conflicts of interest in this work. 


\section{References}

1. Johnson MH. Functional brain development in humans. Nat Rev Neurosci. 2001;2(7):475-483.

2. Johnson MH. Interactive specialization: a domain-general framework for human functional brain development? Dev Cogn Neurosci. 2011;1(1):7-21.

3. Werner H. The Concept of Development from a Comparative and Organismic Point of View. Harris D, editor. Minneapolis, MN: University of Minnesota Press; 1957.

4. Buckner RL, Andrews-Hanna JR, Schacter DL. The brain's default network: anatomy, function, and relevance to disease. Ann N Y Acad Sci. 2008;1124:1-38.

5. Smyser CD, Snyder AZ, Neil JJ. Functional connectivity MRI in infants: exploration of the functional organization of the developing brain. Neuroimage. 2011;56(3):1437-1452.

6. Vogel AC, Power JD, Petersen SE, Schlaggar BL. Development of the brain's functional network architecture. Neuropsychol Rev. 2010;20(4): $362-375$.

7. Thomason ME, Dassanayake MT, Shen S, et al. Cross-hemispheric functional connectivity in the human fetal brain. Sci Transl Med. 2013;5(173):173ra24.

8. Damaraju E, Phillips JR, Lowe JR, Ohls R, Calhoun VD, Caprihan A. Resting-state functional connectivity differences in premature children. Front Syst Neurosci. 2010;4. pii 23.

9. Doria V, Beckmann CF, Arichi T, et al. Emergence of resting state networks in the preterm human brain. Proc Natl Acad Sci US A. 2010; 107(46):20015-20020.

10. Fransson P, Skiöld B, Engström M, et al. Spontaneous brain activity in the newborn brain during natural sleep - an fMRI study in infants born at full term. Pediatr Res. 2009;66(3):301-305.

11. Fransson P, Skiöld B, Horsch S, et al. Resting-state networks in the infant brain. Proc Natl Acad Sci U S A. 2007;104(39):15531-15536.

12. Gao W, Zhu H, Giovanello KS, et al. Evidence on the emergence of the brain's default network from 2-week-old to 2-year-old healthy pediatric subjects. Proc Natl Acad Sci U S A. 2009;106(16):6790-6795.

13. Krueger F, Barbey AK, Grafman J. The medial prefrontal cortex mediates social event knowledge. Trends Cogn Sci. 2009;13(3):103-109.

14. Smyser CD, Inder TE, Shimony JS, et al. Longitudinal analysis of neural network development in preterm infants. Cereb Cortex. 2010;20(12): 2852-2862.

15. Supekar K, Musen M, Menon V. Development of large-scale functional brain networks in children. PLoS Biol. 2009;7(7):e1000157.
16. Fair DA, Dosenbach NU, Church JA, et al. Development of distinct control networks through segregation and integration. Proc Natl Acad Sci U S A. 2007;104(33):13507-13512.

17. McKeown MJ, Hansen LK, Sejnowsk TJ. Independent component analysis of functional MRI: what is signal and what is noise? Curr Opin Neurobiol. 2003;13(5):620-629.

18. Bell AJ, Sejnowski TJ. An information-maximization approach to blind separation and blind deconvolution. Neural Comput. 1995;7(6): 1129-1159.

19. Allen EA, Erhardt EB, Damaraju E, et al. A baseline for the multivariate comparison of resting-state networks. Front Syst Neurosci. 2011;5:2.

20. Shirer WR, Ryali S, Rykhlevskaia E, Menon V, Greicius MD. Decoding subject-driven cognitive states with whole-brain connectivity patterns. Cereb Cortex. 2012;22(1):158-165

21. Marrelec G, Krainik A, Duffau H, et al. Partial correlation for functional brain interactivity investigation in functional MRI. Neuroimage. 2006;32(1):228-237.

22. Salvador R, Suckling J, Coleman MR, Pickard JD, Menon D, Bullmore E. Neurophysiological architecture of functional magnetic resonance images of human brain. Cereb Cortex. 2005;15(9):1332-1342.

23. Ginestet CE, Simmons A. Statistical parametric network analysis of functional connectivity dynamics during a working memory task. Neuroimage. 2011;55(2):688-704.

24. Benjamini Y, Hochberg Y. Controlling the false discovery rate: a practical and powerful approach to multiple testing. J R Stat Soc Ser B. 1995;57(1):289-300.

25. Sporns O. Network attributes for segregation and integration in the human brain. Curr Opin Neurobiol. 2013;23(2):162-171.

26. Kelly AM, Di Martino A, Uddin LQ, et al. Development of anterior cingulate functional connectivity from late childhood to early adulthood. Cereb Cortex. 2009;19(3):640-657.

27. Stevens MC, Pearlson GD, Calhoun VD. Changes in the interaction of resting-state neural networks from adolescence to adulthood. Hum Brain Mapp. 2009;30(8):2356-2366.

28. Fair DA, Cohen AL, Power JD, et al. Functional brain networks develop from a "local to distributed" organization. PLoS Comput Biol. 2009;5(5):e1000381.

29. Heep A, Scheef L, Jankowski J, et al. Functional magnetic resonance imaging of the sensorimotor system in preterm infants. Pediatrics. 2009;123(1):294-300. 


\section{Supplementary materials}

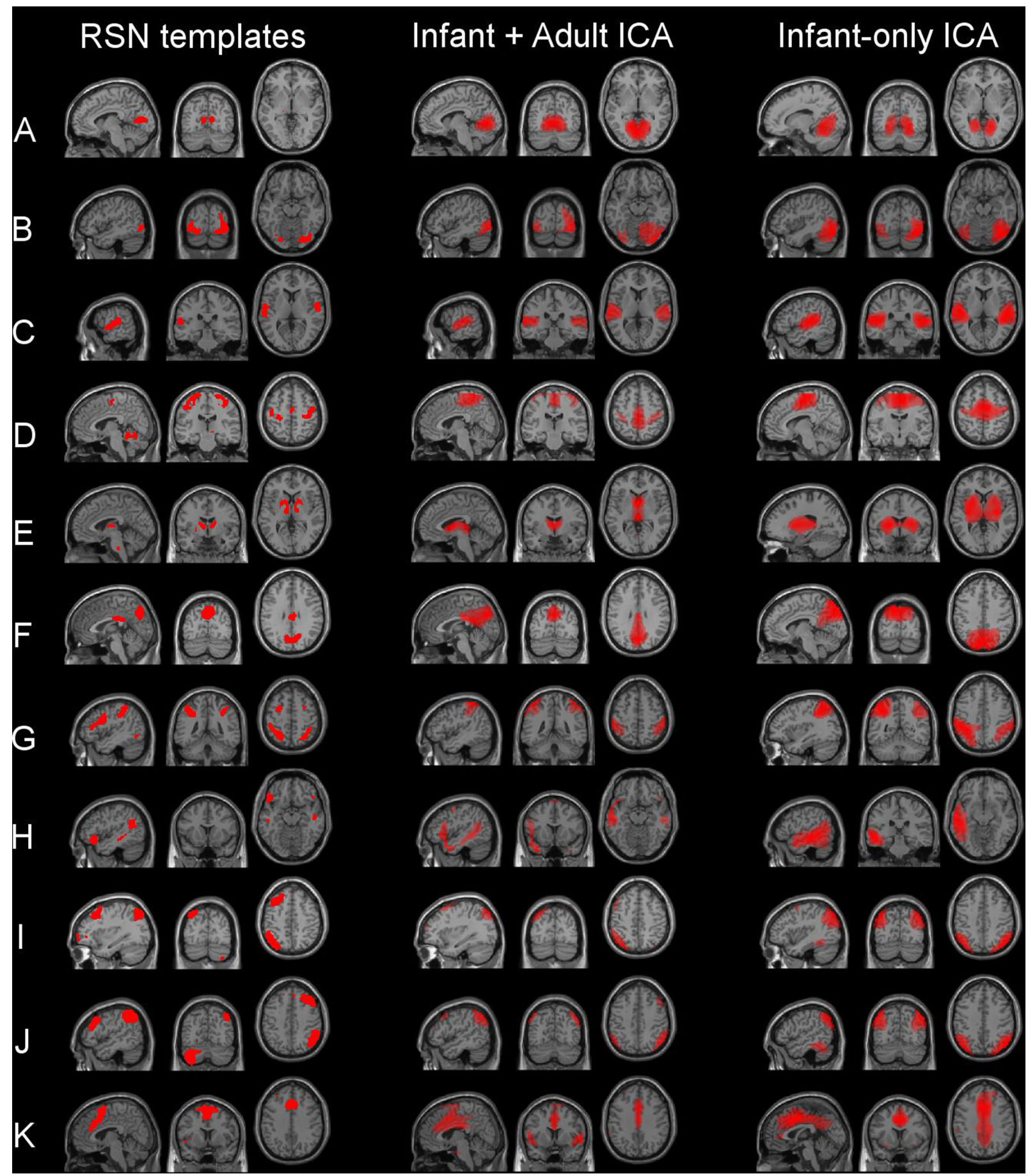

Figure SI Consistency of infant resting-state networks compared with adult template resting-state networks.

Notes: Combined independent components analysis, using both infant and adult subjects, resulted in networks that closely resembled networks from an independent components analysis using the infant subjects only. In both cases, many networks showed spatial distributions matching resting-state network templates.' (A) VI, (B) V2, (C) auditory, (D) sensorimotor, (E) basal ganglia, (F) precuneus, (G) visuospatial, (H) language, (I) left executive control, (J) right executive control, and (K) anterior salience. Abbreviations: RSN, resting-state network; ICA, independent components analysis. 
Table SI Group differences in resting-state networks

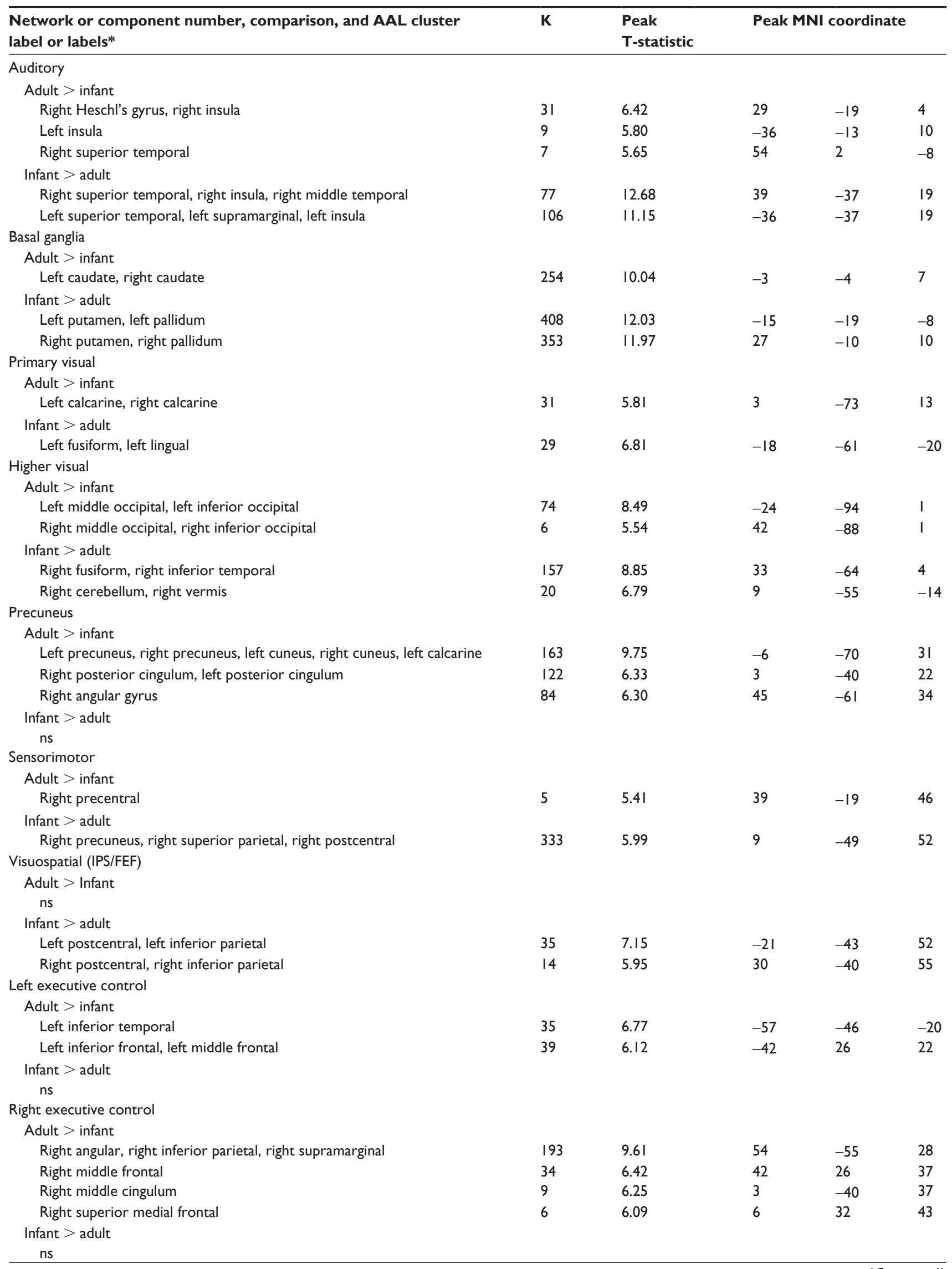


Table SI (Continued)

\begin{tabular}{|c|c|c|c|c|c|}
\hline \multirow{2}{*}{$\begin{array}{l}\text { Network or component number, comparison, and AAL cluster } \\
\text { label or labels* } \\
\text { Anterior salience (insula/dorsal anterior cingulate cortex) }\end{array}$} & \multirow[t]{2}{*}{$\mathbf{K}$} & \multirow{2}{*}{$\begin{array}{l}\text { Peak } \\
\text { T-statistic }\end{array}$} & \multicolumn{3}{|c|}{ Peak MNI coordinate } \\
\hline & & & & & \\
\hline \multicolumn{6}{|l|}{ Adult $>$ infant } \\
\hline Left insula, left inferior frontal & 28 & 8.62 & -36 & 11 & -2 \\
\hline Right insula, right inferior frontal & 46 & 7.30 & 36 & 20 & 4 \\
\hline Right supramarginal, right superior temporal & 18 & 6.73 & 60 & -40 & 34 \\
\hline \multicolumn{6}{|l|}{ Infant $>$ adult } \\
\hline Left rolandic operculum & 21 & 8.87 & -57 & -4 & 7 \\
\hline Right middle cingulum & 13 & 7.40 & 9 & -16 & 25 \\
\hline Right rolandic operculum & 8 & 5.81 & 54 & 5 & 4 \\
\hline \multirow{2}{*}{\multicolumn{6}{|c|}{$\begin{array}{l}\text { Dorsal default mode network (medial prefrontal cortex/posterior cingulate } \\
\text { cortex) }\end{array}$}} \\
\hline & & & & & \\
\hline \multicolumn{6}{|l|}{ Adult $>$ infant } \\
\hline Left precuneus, left posterior cingulum, right precuneus & 64 & 8.04 & -3 & -58 & 25 \\
\hline \multicolumn{6}{|l|}{ Infant $>$ adult } \\
\hline Right medial frontal, right superior frontal & 19 & 7.57 & 12 & 59 & 4 \\
\hline Right anterior cingulum & 7 & 5.72 & 15 & 41 & 4 \\
\hline \multicolumn{6}{|l|}{ Language } \\
\hline \multicolumn{6}{|l|}{$\begin{array}{l}\text { Adult }>\text { infant } \\
\text { ns }\end{array}$} \\
\hline \multicolumn{6}{|l|}{ Infant $>$ adult } \\
\hline Left superior temporal & 10 & 6.18 & -57 & -37 & 13 \\
\hline \multicolumn{6}{|l|}{14} \\
\hline \multicolumn{6}{|l|}{$\begin{array}{l}\text { Adult }>\text { infant } \\
\text { ns }\end{array}$} \\
\hline \multicolumn{6}{|l|}{ Infant $>$ adult } \\
\hline Left insula & 158 & 13.26 & -30 & 8 & -14 \\
\hline Left olfactory & 67 & 9.55 & 0 & 11 & -8 \\
\hline Right insula & 58 & 5.82 & 30 & 11 & -17 \\
\hline \multicolumn{6}{|l|}{18} \\
\hline \multicolumn{6}{|l|}{ Adult $>$ infant } \\
\hline Right frontal inferior Tri. & 35 & 5.26 & 51 & 23 & 28 \\
\hline \multicolumn{6}{|l|}{ Infant $>$ adult } \\
\hline Right parietal inferior & 134 & 9.1 & 57 & -52 & 40 \\
\hline Left parietal inferior & 104 & 8.88 & -54 & -55 & 46 \\
\hline
\end{tabular}

Notes: FWE-corrected $P<0.05$. Network names are according to the labels of the networks in the templates derived from Shirer et al. ${ }^{1}$ The names in parentheses are the names for the networks used in the text, tables, and figures of that same paper. Networks without a parenthetic name have consistent naming between the paper and the downloadable templates. *Labels for significant clusters are determined using the AAL atlas and toolbox. ${ }^{2}$ No significant differences were observed for either adult $>$ infant or infant $>$ adult contrasts for the PSAL and ventral default mode networks, which are not listed in the table.

Abbreviations: K, cluster size in voxels; ns, nonsignificant; FWE, familywise error rate; AAL, automated anatomic labeling; PSAL, posterior salience network; IPS/FEF, intraparietal sulcus and frontal eye fields; Tri, triangularis; MNI, Montreal Neurological Institute.

\section{References}

1. Shirer WR, Ryali S, Rykhlevskaia E, Menon V, Greicius MD. Decoding subject-driven cognitive states with whole-brain connectivity patterns. Cereb Cortex. 2012;22(1):158-165.
2. Tzourio-Mazoyer N, Landeau B, Papathanassiou D, et al. Automated anatomical labeling of activations in SPM using a macroscopic anatomical parcellation of the MNI MRI single-subject brain. Neuroimage. 2002;15(1):273-289.

\section{Publish your work in this journal}

Neuropsychiatric Disease and Treatment is an international, peerreviewed journal of clinical therapeutics and pharmacology focusing on concise rapid reporting of clinical or pre-clinical studies on a range of neuropsychiatric and neurological disorders. This journal is indexed on PubMed Central, the 'PsycINFO' database and CAS,

\section{Dovepress}

and is the official journal of The International Neuropsychiatric Association (INA). The manuscript management system is completely online and includes a very quick and fair peer-review system, which is all easy to use. Visit http://www.dovepress.com/testimonials.php to read real quotes from published authors. 